\title{
Artificial neural networks in slope of road embankment stability applications: a review and future perspectives
}

\author{
Rufaizal Che Mamat $^{1 *}$, Azuin Ramli², Abd Manan Samad ${ }^{3}$, Anuar Kasa ${ }^{1}$, Siti Fatin Mohd Razali ${ }^{1}$ \\ and Mohd Badrul Hafiz Che Omar \\ Department of Civil Engineering, Faculty of Engineering and Built Environment, Universiti Kebangsaan Malaysia, \\ 43600 UKM Bangi, Selangor, Malaysia ${ }^{1}$ \\ Department of Civil Engineering, Politeknik Ungku Omar, Jalan Raja Musa Mahadi, 31400 Ipoh, Perak, Malaysia ${ }^{2}$ \\ Faculty of Architecture, Planning \& Surveying, Universiti Teknologi MARA, 40450 Shah Alam, Selangor, \\ Malaysia $^{3}$ \\ Department of Civil Engineering, Politeknik Sultan Idris Shah, Sungai Lang, 45100 Sungai Ayer Tawar, Selangor, \\ Malaysia $^{4}$
}

Received: 10-October-2020; Revised: 08-February-2021; Accepted: 12-February-2021

(C)2021 Rufaizal Che Mamat et al. This is an open access article distributed under the Creative Commons Attribution (CC BY) License, which permits unrestricted use, distribution, and reproduction in any medium, provided the original work is properly cited.

\begin{abstract}
The artificial or neural network is one of the branches of the artificial intelligence method. Over the last few decades, artificial neural networks (ANNs) have been widely used to predict embankment stability. This paper will provide a detailed review of the ANN application, which is multilayer feedforward neural networks (MLFNN) in road embankment stability. A proposal for further research needs in this area is also discussed. Due to its acceptable accuracy prediction, the ANN model is widely recognized as a successful embankment stability approach. Based on the findings of this paper, it will be able to pave the way for researchers to use the ANN in predicting the stability of road embankment comprehensively.
\end{abstract}

\section{Keywords}

Artificial neural network, Multilayer feedforward neural networks, Road embankment, Slope stability, Prediction.

\section{Introduction}

Ground excavation and land reclamation are essential works in construction activity. In order to ensure the construction structure is safe, the embankment must meet the performance requirements and bear the structure's load. Typically, embankment stability is significant with the settlement because of the consolidation process during and after construction [1-2]. However, surveys found that embankment stability is influenced by settlement [3-12], slope stability [13-20], geotechnical parameters [21-28], and seismic [29, 30]. Although embankment construction is the oldest technology in construction, the design process's engineering challenges are high, especially in predicting settlement [31].

\footnotetext{
*Author for correspondence

This work was supported in part by the Ministry of Higher Education Malaysia (Grant No: FRGS / 1/2018 / TK06 / UITM / 01/1)
}

Inaccurate forecasts will lead to stability problems that can damage construction structures. Today, the settlement is often predicted with uncertainty, using the outcome from monitoring settlement at the initial stages of construction to predict long-term settlement [32]. Most of these methods require a large number of on-site measurements to allow accurate predictions.

Predictions can be conducted using modeling and simulation methods to describe the behavior and produce data and results without practical experiments. The modeling methods are widely used and received in the engineering field. Soil settlement is a crucial parameter in embankment stability. However, existing operational constraints limit the ability to account for it as experiments in the laboratory and on-site are time-consuming and increase project cost. Therefore, it is essential to predict embankment stability using multiple variables. The settlement process is typically highly 
complex so that mathematical models are difficult and inappropriate because of the relationship between the input and the output parameters [33]. The computerized intelligence model provides greater flexibility than statistical models when complex datasets such as non-linear or missing datasets are present. In recent years, artificial intelligence (AI) prediction methods such as artificial neural networks (ANNs) in modeling embankment stability have been used with success. The use of ANN in geotechnical engineering began in 1994 to predict the potential for problematic soil liquefaction [34]. It is a computational and electronic model created based on a biological neural network and able to solve many complex engineering systems.

The ANN represents modeling techniques particularly for datasets with non-linear relationships that do not require any knowledge of data sources but many weights to be estimated and large training sets. Previous surveys were performed on the stability of the embankment and the use of ANN engineering indicated that no relevant studies had recorded the use of ANN to predict the stability of the embankment. Therefore, this study's two primary objectives are (i) to summarise previous studies on the stability of road embankment using the model of ANN, (ii) to present reasons for further research on the application of ANN to stability on embankments.

\section{Artificial neural networks model}

The neural network functions as a brain-like machine to carry out certain tasks. The ANN consists of many processing elements known as neurons or nodes that are interconnected with each other. Neurons interact with each other through weighted connections. The first neural network is trained to process the most matching patterns of inputs and outputs. In addition to predicting output patterns, it can also identify the similarities in new input patterns when presented after training. It also detects similarities in inputs, even when they are not previously known [35]. Therefore, it has excellent interpolation capability even with unclear and complicated input data.

Neural networks can be used for autocorrelation [36], multi-variable regression [37], linear regression [38], trigonometric [39] and various other statistical analysis techniques [40]. Several researchers defined the structure and operation of ANN [41, 42]. As shown in Figure 1, the structure and its operation are formed by three layers consisting of the input layer, hidden layer, and output layer. Data are connected to the network in the input layer, while the output layer holds the network response to the input. The hidden layer allows the network to recognize the more complex characteristics of the input and output. The number of hidden layers depends on the complexity of the pattern recognition problem [43]. Typically, one or two hidden layers are extremely efficient for most problems. The ANN architecture is commonly known as the feed-forward multi-layer perceptron (MLP). It is developed through the creation of a topology network under various combinations of neurons. Once the network is successfully set up, the ANN model will be trained using input and output datasets. The neural network updates the learning algorithms to change their parameters and produces outputs matching the inputs during training. This case is structured to mitigate potential mistakes. The Levenberg-Marquardt (LM), Gauss-Newton (GN), and Gradient descent (GD) are several algorithms. However, LM is often preferred because its convergence is faster than in the GD method, apart from its greater robustness compared to the GN method [44].

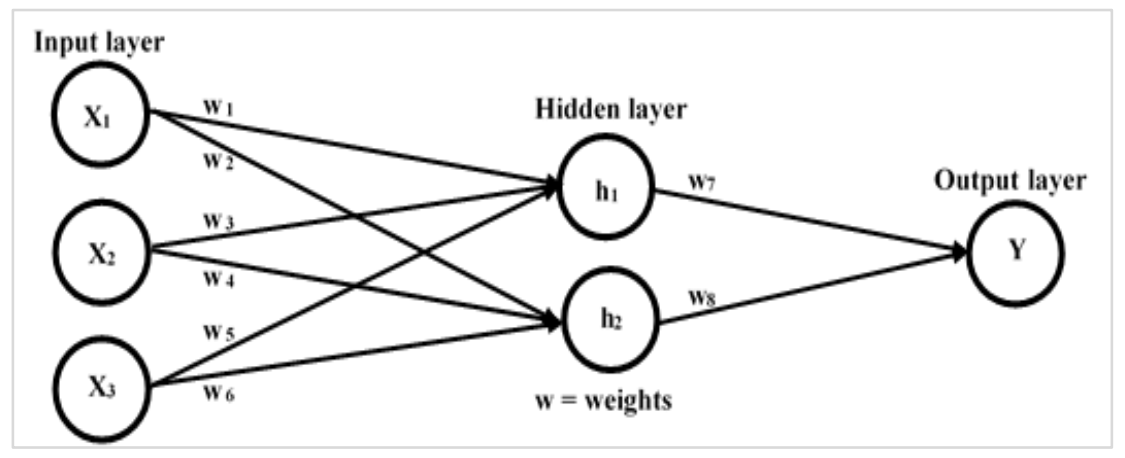

Figure 1 The ANN architecture 
The ANN model can create nonlinear relationships between independently-dependent and experimentally-based variables. In order to build an ANN model, the ANN parameters need to be set beforehand. ANN's crucial parameters include the number of hidden layers, the number of neurons on each layer, the network's configuration, the transfer function, the algorithm training, and the training's optimum rate [45]. The number of neurons in the input layer typically corresponds to the number of analysis parameters, such as slope height, cohesion value, soil angle of internal friction, slope angle, and surcharge. The determination of the numbers of neurons in the output layer typically equals the variables number that predicts the embankment's stability. The number of neurons and hidden layers can be changed to achieve accurate results.

Learning rates will influence the growth in the weight vector step size. In general, learning rates are set during training, in which the optimum learning rates are obtained through the trial-and-error method [46], [47]. It is determined in the optimization context and minimizes the function of neural network loss. It can reach a local minimum of body weight. Momentum is used to accelerate the process. In the backpropagation algorithm, momentum can help accelerate convergence and avoid the local minimum problem. Therefore, an effective combination of learning rate and momentum factor will provide an ideal weight vector during training.

The number of researchers from 2005 until now, who reported using the ANN model in embankment research is shown in Figure 2. This clearly shows the increasing acceptance of ANN's use in the study of embankment stability over the last decade. The studies on embankment stability used the multilayer feedforward neural networks (MLFNN) ANN model.

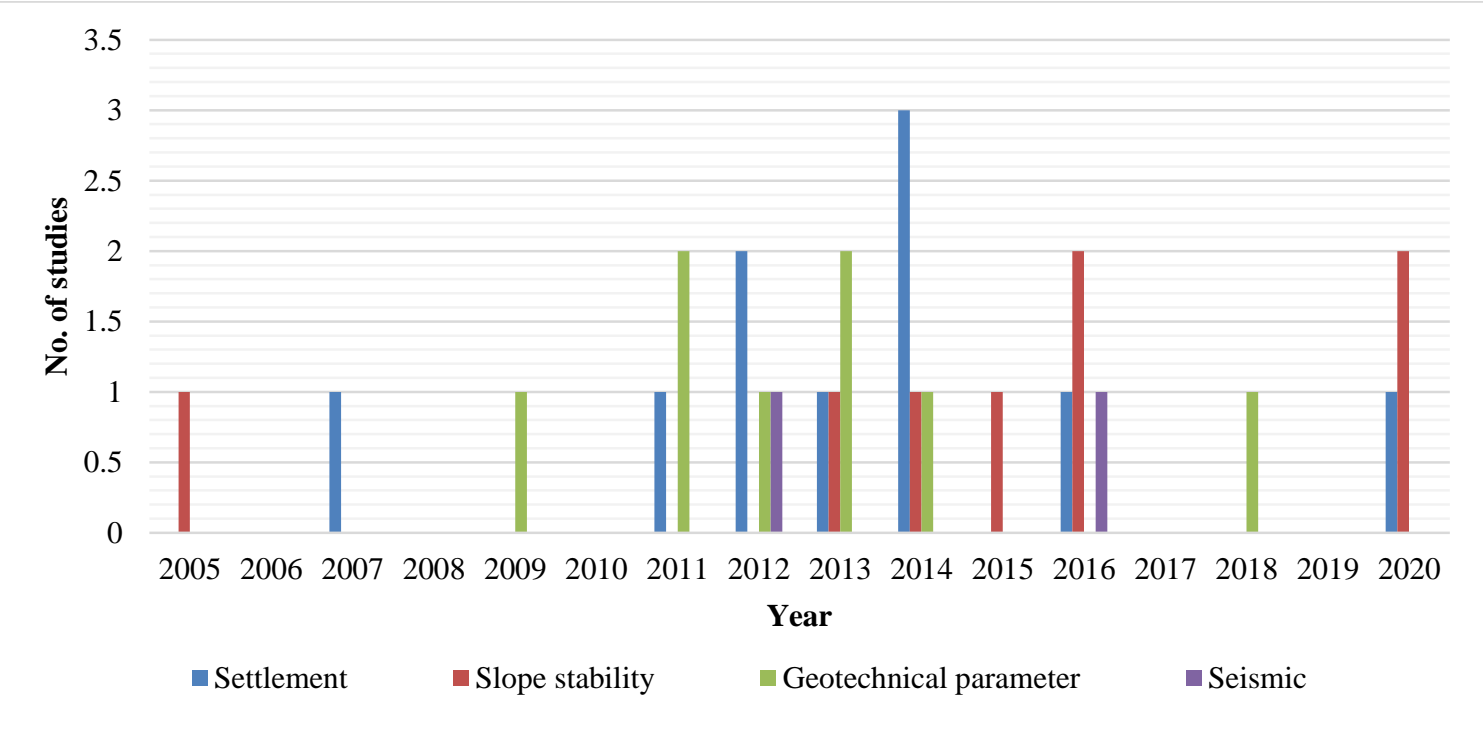

Figure 2 Applications of ANN models for embankment stability

2.1Multilayer feedforward neural networks The threshold logic unit (TLU) is the first ANN. It acts like a "black box" model that applies the human brain's basic conception. The prediction [48], control systems [49], classification [50], optimization [51] and decision-making [52] were widely adopted. This is a popular method in different areas. MLP through ANN, as in Figure 3, is the most popular method compared to others. It can have one or more hidden layers. However, MLP networks with one hidden layer have been proven to provide better predictions [53].
Generally, MLP has the ability to learn through training. This exercise requires one training dataset comprising a series of input and output vectors. During training, the MLP is repeatedly presented with training and weighted data in a predetermined network so that desired input-output mappings occur [54]. The way it learns is supervised. The provided input might not be the same as the expected output. The difference between the expected and actual output is defined as an error signal. The magnitude of the error signal can be determined in order to change the network weights [55]. Therefore, using the 
magnitude of the signal during the exercise can reduce MLP errors.

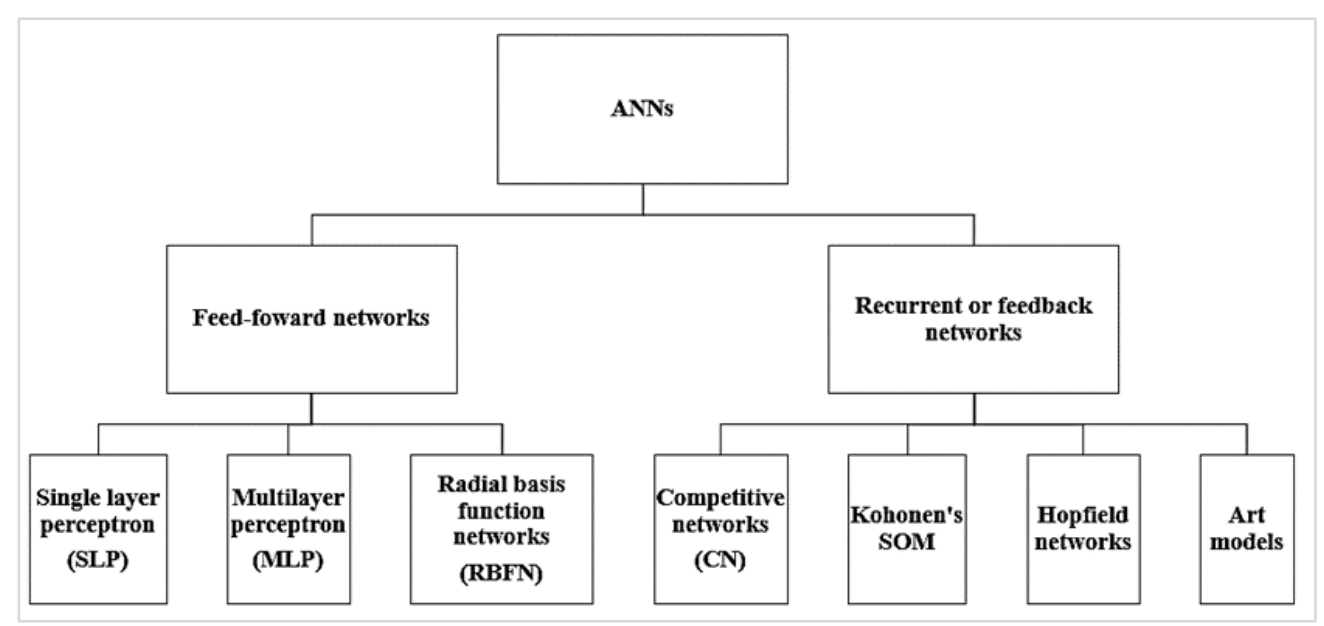

Figure 3 A taxonomy of ANN architectures

\subsection{Training process}

Network training consists of weight and bias modification between neurons through the use of acceptable methods of training and a collection of corresponding output-input data. Data sets can be divided randomly or automatically into three levels, i.e., training, testing, and validation. Training is a set of data used to adjust neuron weight to build models at the beginning stage. This data is important because it should reflect all the problems that occur. It should include all the data owned by the problem domain and be used in the training phase to update the network weights. Test data is used to check the final performance of the developed model. In addition, it is used during the learning process to examine network responses of untrained data. This dataset must be independent of the training and validation datasets. The ANN architecture can be changed, and more training cycles may be used based on performance results. Validation is a step to stop overfitting. This procedure is to examine its weight for accuracy in model predictions. Therefore, the weights cannot be adjusted. However, the training procedures and parameters can be optimized.

The methods of learning are classified into unsupervised learning, evolution, and monitoring [56]. The output is a parameter that relies on the resulting network for a matched input, which is then compared to the expected output. This training is carried out until the network output matches the expected output. A change in weight and bias may reduce the error between the network output and expected output. Since all errors are within the appropriate tolerance or epochs is achieved, the training process will automatically stop.

\subsection{Learning programme}

The backpropagation (BP) neural network is the most frequently-used multi-layered feedforward. The use of BP for training algorithms is very easy. It works by taking the negative direction from the sum of squared errors concerning weight variables to balance and coordinate network weights. Assuming input as $x$ vector, weight as $w$ vector, while network output as $o$ and expected output as $o_{d}$ vector. In Equation (1) the error vector is defined:

$e=o-o_{d}$

The weight in the BP neural network is as in Equation (2).

$w_{t+1}=w_{t}-\eta \frac{1}{2} \frac{\partial e^{t} e}{\partial x}$

where $\eta$ is the learning parameter. However, there are some suggestions to speed up the learning process using the momentum parameter $\mu$ [57]. When it is used, the weight in the BP neural network is in Equation (3).

$w_{t+1}=w_{t}-\eta \frac{1}{2} \frac{\partial e^{t} e}{\partial x}+\mu\left(w_{t}-w_{t-1}\right)$

The LM algorithm is also known as the damped leastsquares method, which is a variation of the Gauss Newton's method. When used in neural network training, its performance index is the mean squared error. In Gauss Newton, the weight of the neural network training is determined as in Equation (4). 
$w_{n+1}=w(n)-\left(J(n)^{T} J(n)\right)^{-1} J(n)^{T} e(n)$

where $J$ is the Jacobi matrix and is defined as in Equation (5).

$J=\left[\begin{array}{ccc}\frac{\partial e_{1}}{\partial w_{1}} & \cdots & \frac{\partial e_{1}}{\partial w_{n}} \\ \vdots & \ddots & \vdots \\ \frac{\partial e_{m}}{\partial w_{1}} & \cdots & \frac{\partial e_{m}}{\partial w_{n}}\end{array}\right]$

Therefore, the transformation of LM to the Gauss Newton's method is as in Equation (6).

$w_{n+1}=w(n)-\left(J(n)^{T} J(n)+\mu I\right)^{-1} J(n)^{T} e(n)$

The $\mu$ ensures that the inverse matrix generates results always and that the value of the sum of the squared error is dependent. When the error reduces, then the $\mu$ parameter is divided by several scalar $\theta$, and vice versa, if the error increases, the parameter $\mu$ is multiplied by $\theta$ scalar. Therefore, the use of values for these parameters should be in line with network learning.

The transfer function is applied to the weighted input of neurons to produce the final output. This function highly affects the performance of neural networks because neural networks cannot learn about nonlinear relationships [58]. Three transfer functions are often used for multilayer networks, as shown in Table 1. The sigmoid function curve is S-shaped, as in Figure 4(a). The main reason for the use of a sigmoid function is its value of between 0 and 1 . Probability lies only between the range of 0 and 1 . Therefore, it is suitable to be used on the model to predict the probability of the output. This function can be distinguished by finding the slope of the sigmoid curve at any two points. It can also lead to stuck on the neural network during training. The use of the hyperbolic tangent function is one of the alternative approaches to addressing the problem. The tanh function range is between -1 and 1 . The tanh function graph is also $S$-shaped, as shown in Figure 4(b). Negative inputs will be mapped strongly negative, while zero input will be mapped approaching zero on the tanh graph. Most real models have non-linear input or output features, but there are several models, when operated in nominal parameters, display a linear behavior. Such behavior is acceptable to the purelin transfer function, as in Figure 4(c).

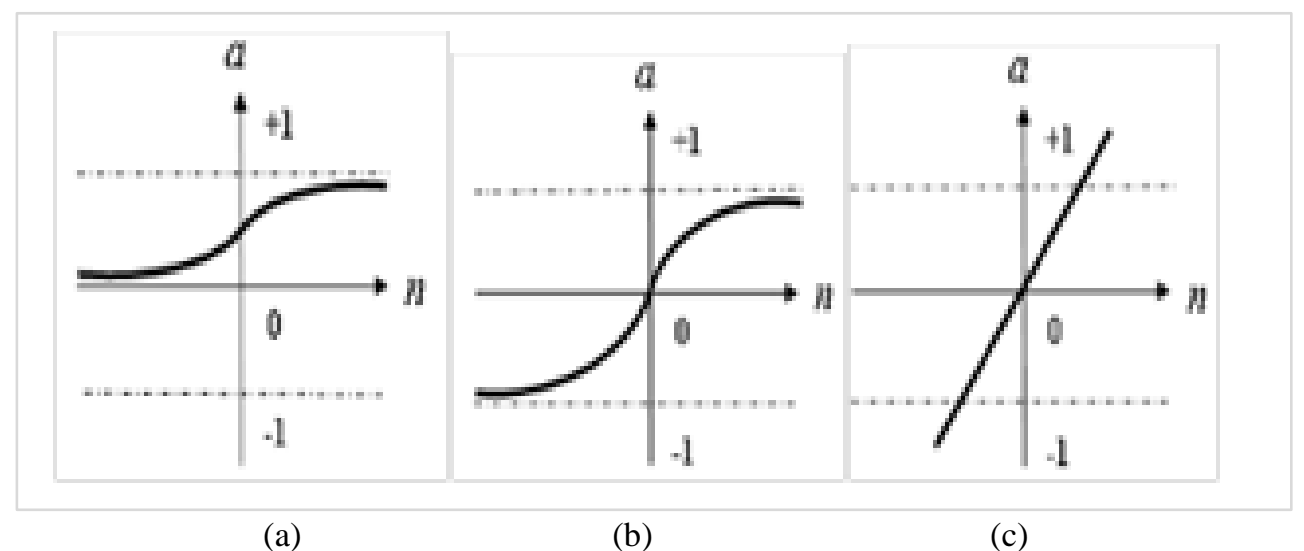

Figure 4 The transfer functions: (a) log-sigmoid (b) tan-sigmoid (c) purelin

The transfer function can be divided into two types, linear and non-linear. Linear or identity transfer function, such as in Figure 5(a), has output function which will not be enclosed between ranges. With this unconnected range, it does not help in neural network problems. Therefore, it is rarely used by researchers. The non-linear transfer function, on the other hand, is the most widely used activation function. It helps to produce the graph as in Figure 5(b), apart from its adaptability to different types of data and its ability to distinguish between outputs.
The most important, yet time-consuming step is the data collection process. In neural network learning, data must be obtained in the correct format [59]. The data formatting process is known as normalization. Input data is highly dependent on the transfer function. Normally the data should lie in the [0.1] or $[-1,1]$ interval if the neuron activation function is sigmoid or tanh. If normalization is not used, data input will give additional effect. The use of multiple data in training can result in overfitting. Normalization can speed up training and reduce stuck 
in local optimum. Therefore, normalizing can ensure that data is uniformly distributed between the network input and the output and can eliminate some data vector dimensions' geometric bias.

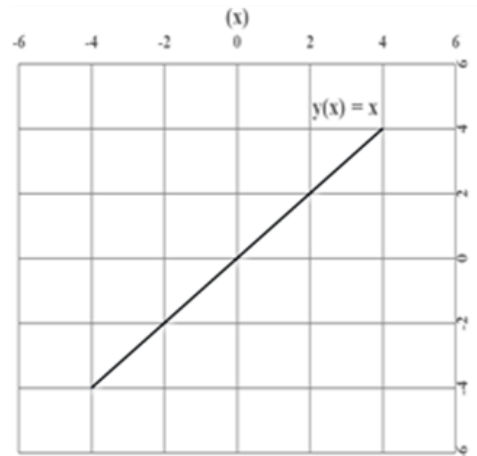

(a)

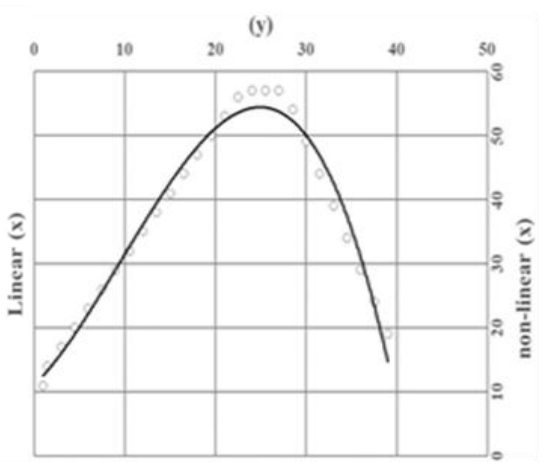

(b)

Figure 5 The types of transfer function: (a) linear (b) non-linear

Table 1 Performance values are obtained by evaluating processes from the algorithm

\begin{tabular}{lll}
\hline Functions & Transfer function & Derivatives of the transfer function \\
\hline Logistic sigmoid (LS) & $y(x)=\frac{1}{1+\exp (-x)}$ & $y^{\prime}(x)=y(x)(1-y(x))$ \\
\hline Hyperbolic tangent (HT) & $y(x)=\frac{2}{1+\exp (-2 x)}-1$ & $y^{\prime}(x)=1-y(x)^{2}$ \\
\hline Linear function (LF) & $y(x)=x$ & $y^{\prime}(x)=1$ \\
\hline
\end{tabular}

The two commonly used methods for normalization are the min.-max. and the normal distribution methods. The min-max method assigns original values linearly to a new interval, as shown in Figure $6(a)$. The original minimum value $x \mathrm{~min}$ and maximum value $x \max$ can be obtained from raw statistical data, while new minimum value $y$ min and maximum value $y$ max is given by researcher, then the $x$ value can be calculated from the original $x$ value such as in Equation (7). The original values are mapped by the new mean and the deviation in a normal distribution, as shown in Figure 6(b). The y value can be calculated from equation as in Equation (8).

$y=\frac{\left(x-x_{\min }\right) \cdot\left(y_{\max }-y_{\min }\right)}{x_{\max }-x_{\min }}+y_{\min }$

$y=\frac{\left(x-x_{\text {mean }}\right) \cdot y_{\text {std }}}{x_{\text {std }}}+y_{\text {mean }}$

Following are the steps of the ANN training process:

i. The data set should be collected from the experiment and the variables that affect the performance of the embankment stability need to be identified. ii. The input and output variables are specified.

iii. Input and output data are normalized in the range between 0 and 1 ; between -1 and 1 or 0.1 and 0.9 as in Equation (7) and Equation (8). Normalization of input and output data will increase the network training rate.

iv. Randomly collected data is divided into two datasets, namely training and testing datasets. The training dataset is used to produce the model output while the testing dataset is used to check the trained ANN parameters. About $70-80 \%$ of the randomly selected datasets are used as training datasets while the remaining data can be used to test the model.

v. The ANN model is developed and trained to optimize ANN model parameters for high accuracy.

vi. The choice of the best ANN model is based on the results of the optimally trained model and the performance criteria. 


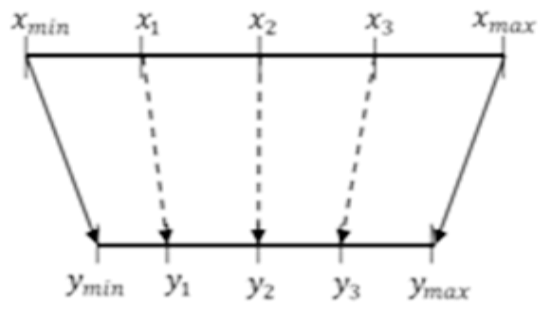

(a)

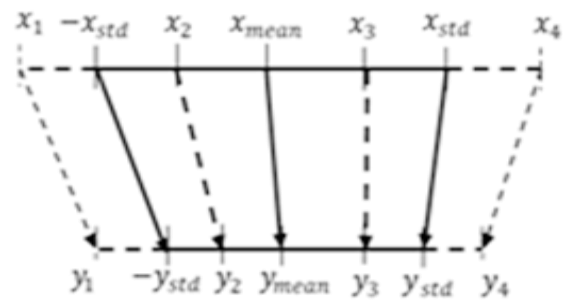

(b)

Figure 6 The types of normalization method: (a) min.-max. (b) normal distribution

\subsection{Statistical parameters}

Generally, the model's performance is evaluated by comparing calculated output with actual data. The models of performance are assessed by statistical parameters, i.e. the coefficient of determination (R2), average relative error (ARE), correlation coefficient $(\mathrm{R})$, the root mean square values (RMSE), Chi-square statistic $(\chi 2)$, mean absolute error (MAE), average error (AE), value account for (VAF), mean absolute relative error (MARE), and mean squared error (MSE) by altering the model parameters. To obtain good results, the model parameters should be optimized using experimental methods or the novel optimization algorithm [60, 61]. A high-performance index in training datasets indicates a successful predictive modeling process while a highperformance index in testing datasets indicates a satisfactory generalization capability of the model.

The coefficient of determination as in Equation (9) is also known as the multiple correlation coefficient and is formed during the classical regression analysis. It is defined as the proportional variance by regression model which is useful as a measure of success to predict dependent variables from independent variables. The $\mathrm{R} 2$ value of 1 is the best model. A higher R2 value indicates better and more precise predictive model. ARE as in Equation (10) is the standard deviation of the sample average. It reflects the average difference level from the sample and population mean. Usually, R or known as Pearson's R as in Equation (11) is used in statistics to measure the level of strength of the linear relationship between two variables. The range of values for this correlation coefficient is between -1.0 and 1.0. A correlation coefficient greater than 1.0 or less than -1.0 indicates an incorrect correlation measure. The RMSE as in Equation (12) is also known as the quadratic mean and is a benchmark of the difference in value based on observable values. Lower RMSE indicates a more accurate predictive model. If the value of RSME is 0 then it is defined as a very good model. Chi-square 310 statistic as in Equation (13) is used to investigate whether the distribution of category variables differ from one another.

$$
\begin{aligned}
& R^{2}=1-\frac{\sum_{i=1}^{N}\left(y_{\text {prd. } i}-y_{\text {exp. } i}\right)^{2}}{\sum_{i=1}^{N}\left(y_{\text {prd. } i}-y_{m}\right)^{2}} \\
& A R E=\frac{100}{N} \sum_{i=1}^{N}\left|\frac{y_{\text {exp. } i}-y_{\text {prd. } . i}}{y_{\text {exp } . i}}\right| \\
& R=\sqrt{1-\frac{\sum_{i=1}^{N}\left(y_{\text {prd. } .}-y_{\text {exp. } . i}\right)^{2}}{\sum_{i=1}^{N}\left(y_{\text {prd. } .}-y_{m}\right)^{2}}} \\
& R M S E=\sqrt{\frac{\sum_{i=0}^{N}\left(y_{\text {prd. } .}-y_{\text {exp. } .}\right)^{2}}{N}} \\
& \chi^{2}=\sum_{i=1}^{N}\left[\frac{\left(y_{\text {prd. } i}-y_{\text {exp. } . i}\right)^{2}}{y_{\text {prd.i }}}\right]
\end{aligned}
$$

In addition, it can also compare the similarities in categories between two or more independent groups. MAE as in (14) is a common measurement of prediction error in time series analysis. It is a more reliable goodness-of-fit for moderate values. Its size can be used to determine the suitability of the resulting output according to the required output. MAE value of 0 indicates the best ANN model. In addition, performance indexes such as ARE, AE, VAF and MARE take percentage values, whereby a value of 100 indicates the best model. MSE as in (18) is a measure of performance quality of the estimator or the predictor that is always non-negative, and the value closer to zero is better. The size of the MSE requires a predicted target or an estimate together with the target or estimator that is a function of the data provided.

\section{Modelling of road embankment stability}

Road embankment stability includes several geotechnical aspects that often considered in the assessment, namely ground settlement, slope stability, soil characteristics, seismic influence, load influence, pore water pressure influence, and ground deformation [62]. In this section, the prediction of ground settlement, slope stability, geometric 
parameters, and earthquake influence using ANN is reviewed and shown in Table 2. In the relevant work, Chik et al. [3] used a wide range of geometric parameters as inputs in the ANN model to predict ground settlement under the embankment. In contrast, Kanayama et al. [4] use three input parameters, namely consolidation period, height, and consolidation stages, to predict the sedimentation of the soil under the embankment. In the study conducted by Chik and Aljanabi [5], soil properties and various geometric features of the embankment were used as input parameters of the ANN model to predict the soft ground settlement beneath embankment stabilized with the stone column. In the same field of study, Bi and Ding [6] used a consolidation period representing input parameters to predict the ground settlement under the Jieyang highway embankment. It is clear from this report that the consolidation periods time, embankment geometric characteristics and soil properties are input parameters that need to be considered in predicting settlement.

$\mathrm{Li}$ and $\mathrm{Bu}$ [7] developed the ANN model with a 4-51 configuration to predict soft ground settlement. By using a learning rate of 0.025 , the prediction performance produced is high. Also, On the other hand, Wang et al. [8] found that the backpropagation network has a slow in training speed and problematic the local minimum. Hasanzadehshooiili et al. [9] used a three-layer backpropagation network to predict sandy gravel settlement beneath the embankment. In his study, network structures with 4-6-2 and 4-9-2 were selected with the sigmoid function used as a transfer function for all network layers. The performance results, as shown in Table 3, can be concluded this network structure can be well predicted and considered for various geotechnical engineering studies. Kurnaz et al. [10] developed the ANN three-layer backpropagation model with a 420-2 network configuration. In his study, the neuron number in the hidden layer was determined by a trial and error method is successful with a range of neurons 8 to 40 . The performance of models with optimal network structure found R2 values for training and testing of 0.96 and 0.94 , respectively. This clearly shows the tried and tested method applied to determine the optimal network to function properly.

Dinçer [11] developed the ANN network structure with a 4-4-1 configuration to predict the modulus of deformation and subgrade reaction coefficients for the earth-filling structure. Based on the performance index shown in Table 4, the $\mathrm{R} 2$ value generated for EV1 is better than other parameters. This clearly shows that the data pattern for EV 1 can be learned well. Data patterns play an important role in the ANN model to produce high accuracy.

Table 2 A summary of investigations on modeling of embankment stability

\begin{tabular}{|c|c|c|c|}
\hline Stability features & Optimal number of neurons & Evaluation indices & References \\
\hline Settlement & $5-4-1$ & $\mathrm{R}=0.997$ & [3] \\
\hline Settlement & $3-10-1$ & $\mathrm{CV}<1$ and $2 \%$ & [4] \\
\hline Settlement & $4-15-1$ & $\mathrm{R}^{2}=0.9315$ & [5] \\
\hline Settlement & $1-2-1$ & $\mathrm{ME}=1.5$ to $3.6 \%$. & [6] \\
\hline Settlement & $4-5-1$ & $\mathrm{SSE}=0.0368$ & [7] \\
\hline Settlement & $7-3-2$ & No information & [8] \\
\hline Settlement & $4-9-2$ & $\mathrm{R}^{2}=0.983, \mathrm{RMSE}=0.082$ & [9] \\
\hline Settlement & $4-20-2$ & $\begin{array}{l}\mathrm{R}_{(\text {train) }}^{2}=0.8926 \\
\mathrm{R}_{\text {(test) }}^{2}=0.8973\end{array}$ & {$[10]$} \\
\hline Settlement & $4-4-1$ & $\begin{array}{l}\mathrm{R}^{2}=0.75, \quad \mathrm{RMSE}=0.72, \\
\mathrm{MAPE}=7.25, \mathrm{VAF}=75.41 \%\end{array}$ & [11] \\
\hline Settlement & $7-36-1$ & $\mathrm{R}=0.9853, \mathrm{MSE}=20.062$ & {$[12]$} \\
\hline Slope & $5-12-12-1$ & $\begin{array}{l}\mathrm{R}=0.996, \quad \mathrm{RMSE}=0.035 \\
\mathrm{MAE}=0.014\end{array}$ & [13] \\
\hline Slope: & & MSE $=\quad 0.01$ (initial data), & [14] \\
\hline Circular failure & $6-6-1$ & $\mathrm{MSE}=0.00003$ (filtered data) & \\
\hline Wedge failure & $8-8-1$ & $\mathrm{MSE}=0.00004$ & \\
\hline Slope & $3-4-1$ & $\begin{array}{ll}\mathrm{R}=0.524, & \mathrm{RMSE}=0.375 \\
\mathrm{MAE}=0.315 & \end{array}$ & {$[15]$} \\
\hline Slope & $5-4-1$ & $\begin{array}{l}\mathrm{R}_{\text {(train) }=0.9975,} \quad \mathrm{R}_{\text {(test) }}^{2}= \\
0.9973, \mathrm{R}_{\text {(valid) }}^{2}=0.9949\end{array}$ & [16] \\
\hline Slope & $4-5-1$ & $\begin{array}{l}\mathrm{R}_{\text {(train) }}^{2}=0.9904, \quad \mathrm{R}_{\text {(test) }}^{2}= \\
0.9837, \mathrm{R}_{\text {(valid) }}^{2}=0.9806\end{array}$ & [17] \\
\hline
\end{tabular}


Rufaizal Che Mamat et al.

\begin{tabular}{|c|c|c|c|}
\hline Stability features & Optimal number of neurons & Evaluation indices & References \\
\hline Slope & $3-5-1$ & $\begin{array}{l}\mathrm{R}=0.997, \quad \mathrm{MAPE}=1.522, \\
\mathrm{MAE}=0.041, \mathrm{NMSE}=0.0073\end{array}$ & [18] \\
\hline Slope & $4-3-1$ & $\mathrm{R}=0.973, \mathrm{MAPE}=1.04$ & [19] \\
\hline Slope & $3-6-1$ & $\mathrm{R}^{2}=0.9999, \mathrm{RMSE}=0.0107$ & [20] \\
\hline Geotechnical parameter & $4-4-1$ & $\begin{array}{ll}\mathrm{R}=0.87, & \mathrm{RMSE}=2.35, \\
\mathrm{MARE}=5.75 & \end{array}$ & [21] \\
\hline Geotechnical parameter & $6-8-8-1$ & $\mathrm{R}_{\text {(train) }}=0.908, \mathrm{R}_{\text {(test) }}=0.862$ & [22] \\
\hline Geotechnical parameter & $2-20-2$ & $\mathrm{R}_{\text {(train) }}^{2}=0.99, \mathrm{R}_{\text {(test) }}^{2}=0.93$ & [23] \\
\hline Geotechnical parameter & $2-120-1$ & $\begin{array}{l}\mathrm{R}=0.98, \mathrm{MAE}_{\text {(train) }}=2.6448, \\
\mathrm{MAE}_{(\text {valid) }}=5.3366\end{array}$ & {$[24]$} \\
\hline Geotechnical parameter & $6-2-2$ & $\begin{array}{lr}\mathrm{R}_{\text {(train) }}=0.940, & \mathrm{R}_{\text {(test) }}=0.879, \\
\mathrm{RMSE}_{\text {(train) }}= & 0.103, \\
\mathrm{RMSE}_{\text {(test) }}=0.137 & \end{array}$ & [25] \\
\hline Geotechnical parameter & $3-3-1$ & $\begin{array}{ll}\mathrm{R}=0.91, & \mathrm{RMSE}=0.019, \\
\mathrm{MAE}=0.025 & \end{array}$ & [26] \\
\hline Geotechnical parameter & $5-5-8-1$ & $\mathrm{R}^{2}=0.9917, \mathrm{RMSE}=0.037$ & [27] \\
\hline Geotechnical parameter & $3-16-1$ & $\begin{array}{l}\mathrm{R}^{2}=0.946, \quad \mathrm{RMSE}=0.79, \\
\mathrm{MAPE}=12.4, \mathrm{VAF}=94.55 \%\end{array}$ & [28] \\
\hline Seismic & $5-10-1$ & $\begin{array}{ll}\mathrm{R}_{\text {(train) }}=0.921, & \mathrm{R}_{\text {(test) }}=0.915, \\
\mathrm{RMSE}_{\text {(train) }}= & 0.061, \\
\mathrm{RMSE}_{\text {(test) }}=0.057 & \end{array}$ & [29] \\
\hline Seismic & $7-5-1$ & $\begin{array}{l}\mathrm{R}_{\text {(train) }}^{2}=0.986, \mathrm{R}_{\text {(test) }}^{2}=0.986, \\
\mathrm{R}_{\text {(valid) }}^{2}=0.985\end{array}$ & {$[30]$} \\
\hline
\end{tabular}

Table 3 Performance of ANN models [9]

\begin{tabular}{lll}
\hline ANN architecture & $\mathbf{R}^{2}$ & RMSE \\
\hline $4-6-2$ & 0.981 & 0.0171 \\
\hline $4-9-2$ & 0.983 & 0.082 \\
\hline
\end{tabular}

Table 4 ANN architecture and performance indices of models [11]

\begin{tabular}{lllll}
\hline Output model & $\mathbf{R}^{\mathbf{2}}$ & RMSE & MAPE & VAF [\%] \\
\hline EV1 & 0.69 & 1.17 & 11.36 & 68.99 \\
\hline EV2 & 0.75 & 0.72 & 7.25 & 75.41 \\
\hline$k_{s}$ & 0.43 & 69.95 & 18.75 & 43.05 \\
\hline
\end{tabular}

Chok et al. [13] found that the ANN structural network with 5-12-12-1 produces the lowest error, where the RMSE and MAE values are 0.035 and 0.014 , respectively. By adapting the tried and error method of determining the total number of neurons and the number of hidden layers, the predictive performance generated to predict the reliability of a heterogeneous cohesive slope is astounding. These findings indicate that the number of layers and neurons has a significant influence on the performance of the ANN model. In another study, Sakellariou and Ferentinou [14] found the three-layer ANN model to be more prestigious for predicting slope stability. With traincgf as a training function on 6-6-1 network structures for circular failure and 8-8-1 for wedge failure, simulation results show that the forecast performance produced is good with measurement data and variance values are also low. 312
This indicates that no one dominant network structure can be used in all road embankment stability prediction applications.

In another study of slope stability predicting, Mamat et al. [15] use geometric features as input parameters. The number of hidden layered neurons is determined by trying and succeeding with a range of 1 to 20 . The results of the training performance performed on $70 \%$ of 100 data sets found that the optimum number of hidden layered neurons is 4. By using the optimal network structure of 3-4-1, MAE, and RMSE values are very low. This indicates that the optimal network structure gives excellent predictive results. Erzin and Cetin [16], on the other hand, found that a 5-4-1 configuration to predict the critical safety factor of a homogeneous slope produces the best prediction compared to other network structures. In his study, 
soil characteristics and slope geometry were used as input parameters. Data sets for training, testing and validation were obtained from the simulation results of the limit equilibrium method. Based on Table 5, the researchers have developed two models, namely ANN-1 and ANN-2, respectively, with the optimum number of hidden layer neurons is five and four. It can be observed that the optimal number of neurons in the hidden layer yields a high predictor. It's clearly, a large number of neurons can help models to read data patterns well.

In the same study, Erzin and Cetin [17] found that a 4-5-1 configuration was able to predict slope stability caused by earthquake strength with high accuracy. The optimal number of hidden layered neurons is determined by $2 \mathrm{I}+1$, where $\mathrm{I}$ is the total of the variables in the input layer. Based on the study results shown in Table 6, the network structure using the procedure resulted in higher predictive performance than the validation and testing data sets. It is clear that this procedure is very useful for determining the best network structure and can reduce the uncertainty encountered during soil engineering projects. Therefore, this procedure can provide a new approach to minimize the potential for correlation inconsistencies.
Abdalla et al. [18] found that a network of structures with a 6-4-1 configuration produced the best predictive accuracy compared to the others. The number of hidden layered neurons is determined using trial and error methods. The hyperbolic tangent transfer function is used on the hidden layer and the linear transfer function on the output layer. Table 7 shows the ANN MLP developed using different sets of geometry and shear strengths based on four wellknown performance evaluation methods, namely Fellenius, Bishop, Janbu, and Spencer. Based on these results, it can be explained that this ANN model is a reliable calculation tool for predicting the stability of clay slopes.

Verma et al. [19] used the ANN model to predict the stability of road embankment slopes. Based on the training performance performed, the ANN model with 4-3-1 configuration achieved the lowest error using the LM algorithm and network function shown in Table 8. The simulation results, MAPE, and R values generated in the training process are 1.04 and 0.973, respectively. This clearly shows that the ANN model can be successfully implemented to predict road slopes' stability accurately.

Table 5 Performance indices of the ANN models developed [16]

\begin{tabular}{llllll}
\hline Model & Data & $\mathbf{R}^{\mathbf{2}}$ & RMSE & MAE & VAF [\%] \\
\hline \multirow{3}{*}{ ANN-1 } & Training set & 0.9975 & 0.07 & 0.05 & 99.75 \\
\cline { 2 - 6 } & Testing set & 0.9973 & 0.07 & 0.05 & 99.73 \\
\cline { 2 - 6 } & Validation set & 0.9949 & 0.07 & 0.06 & 99.49 \\
\hline \multirow{2}{*}{ ANN-2 } & Training set & 0.9915 & 0.09 & 0.12 & 99.15 \\
\cline { 2 - 6 } & Testing set & 0.9911 & 0.09 & 0.12 & 99.11 \\
\cline { 2 - 6 } & Validation set & 0.9903 & 0.07 & 0.10 & 99.01 \\
\hline
\end{tabular}

Table 6 Performance indices (R2, RMSE, MAE, and VAF) of the ANN models developed [17]

\begin{tabular}{lllll}
\hline Data & $\mathbf{R}^{\mathbf{2}}$ & RMSE & MAE & VAF [\%] \\
\hline Training set & 0.9904 & 0.02 & 0.06 & 99.04 \\
\hline Testing set & 0.9837 & 0.03 & 0.08 & 98.36 \\
\hline Validation set & 0.9806 & 0.03 & 0.09 & 98.06 \\
\hline
\end{tabular}

Table 7 Performance indices of ANN model on the test data [18]

\begin{tabular}{lllll}
\hline Methods & R & MAPE & MAE & NMSE \\
\hline Ordinary & 0.99598 & 1.681 & 0.04396 & 0.00908 \\
\hline Janbu & 0.99597 & 1.841 & 0.04595 & 0.00832 \\
\hline Bishop & 0.99664 & 1.522 & 0.04056 & 0.00727 \\
\hline Spencer & 0.99596 & 1.750 & 0.04413 & 0.00888 \\
\hline
\end{tabular}


Rufaizal Che Mamat et al.

Table 8 Parameters for network [19]

\begin{tabular}{ll}
\hline Parameter & Values \\
\hline Learning parameters & 4 \\
Learning rate & 0.1 \\
Momentum parameters & 0.71 \\
Momentum factor & 0.018 \\
Targeted error & 0.001 \\
Networks training function & Trainoss \\
Activation (transfer) function for all layers & Tansig \\
No. of datasets & 100 \\
No. of training sets & 90 \\
No. of testing sets & 10 \\
\hline
\end{tabular}

Kayadelen et al. [21] developed two ANN models to predict the value of the effective shear resistance angle. The network structures of ANN I and ANN II models are 4-5-1 and 4-4-1, respectively, using the sigmoid function as the transfer function. Based on the forecast performance, ANN II produced a lower MARE value of 5.75 compared to ANN I at 6.79. Besides, ANN II showed better predictive ability by producing a maximum absolute $\mathrm{RE}$ value of 13.68. Comparative analysis of the two models' performance showed that ANN II exceeded ANN I significantly, as evidenced by a number of statistical performance criteria used in previous studies.

The ANN model was developed by Park [22] with a 6-8-8-1 configuration to predict soil permeability. Input parameters include natural water content, specific gravity, gravel weight percentage, and sand weight percentage. The tan-sigmoid function is used in the input layer to the first hidden layer, while the log-sigmoid function is used in the first hidden layer to the second hidden layer, and the linear function is applied in the second hidden layer to the output layer. As a result, the performance performed found that the correlation was perfect for training $(\mathrm{R}=0.908)$ and test data $(\mathrm{R}=0.862)$. It clearly shows that the developed ANN model can serve as a simple and reliable predictive tool for predicting soil permeability coefficient without excessive field testing.

Islama et al. [23] used the ANN model to predict the relationship between compacted soil dry density and soil electrical resistance based on experimental data in soil profiles. This developed model uses a 2-20-2 configuration, in which two input neurons include ground electrical resistance and moisture content. Based on the performance analysis performed, the regression relationship between the ANN output value and the target value indicates that the value of $\mathrm{R}^{2}$ is 0.99 for training, while for testing, $\mathrm{R}^{2}$ is 0.93 . 314
This clearly shows that this developed ANN model is reliable with superior predictive performance.

Viji et al. [24] have developed the ANN model to predict fly ash's compaction characteristics. The neurons and the number of hidden layers are determined by trying and succeeding by training several network structures. Based on training performance analysis, 120 neurons were used with one hidden layer giving the best results. The logistic sigmoid transfer function is used for all layers with a linear function applied to the output layer. The study results found that the ANN model can produce accurate predictions and is potentially used for engineering applications compared to the classic regression model.

Kanungo et al. [25] have developed an ANN model with a 5-16-2 configuration to predict unsaturated soil shear strength parameters. The LM algorithm is used to train neural networks, while the log-sigmoid type transfer function is used in the input and output layers. In this study, the ANN model's predictive performance was compared with the Regression Tree (CART) model. The results of this study found that ANN performed better than CART. This clearly shows that ANN can make predictions with higher accuracy than other methods. In another study conducted by Khanlari et al. [26], the ANN model was developed using MLP, and radial basis (RBF) functions to predict shear strength parameters. Upon analysis, it was found that the ANN-MLP model performed better than the ANN-RBF model. This indicates that the network with MLP function is the most suitable and has a significant effect on improving the predicting accuracy.

Ghorbani and Hasanzadehshooiili [27] have developed several ANN models to predict the compressive strength and bearing ratio of California soft ground stabilized with micro silica-lime sulfate. 
After evaluating all network structures, the BP-ANN model with a 5-5-8-1 network configuration is best for predicting as it shows high accuracy results. It also reports that this network configuration produces superior accuracy with $\mathrm{R}^{2}$ and RMSE performance indices of 0.9917 and 0.037 , respectively. The authors also conclude that BP-ANN is a tool capable of predicting effectively and efficiently, even under varying conditions. Yilmaz and Kaynar [28] have developed two different ANN architectures, namely MLP and RBF, to predict the percentage of soil swelling. The number of neurons in the hidden layer is determined using a trial and error method with a range of 1 to 20 neurons. Based on training performance, the optimal ANN-MLP and ANN-RBF configurations are 3-2-1 and 3-16-1, respectively. As can be seen in Table 9, the ANN-RBF model shows a high-performance forecast compared to ANN-MLP.

Gordan et al. [29] used ANN to predict slope stability with seismic effects. This study found that structural networks with 5-10-1 configurations resulted in the best training performance. This study shows that the R2 values for training and testing are 0.921 and 0.915 , respectively. Besides, the use of LM algorithms in this model network has a good effect on optimizing network structure in ANN training. In a study conducted by Erzin and Cetin [30], the ANN and MR models were developed to predict homogeneous finite slopes with earthquake strength. A total of five input parameters were used in this study, namely height, slope angle, cohesion, friction angle, bulk unit weight, distance epicenter, and earthquake magnitude. The optimal ANN network structure is obtained with a 5-5-1 configuration, and the momentum factor is 0.001 . The log-sigmoid transfer function is used to achieve the best performance in training and testing. Based on Table 10 , the ANN model shows that this structure is easily and efficiently trained.

In a study conducted by Gao et al. [20], an ANN model with an imperialist competition algorithm (ICA) was developed to predict the slope stability behaviors that are problematic in slope stability design charts. Using trial and error methods, the optimal network structure for the developed model is 3-6-1. As a result, this model shows very impressive performance with R2 and RMSE values are 0.999 and 0.0107, respectively. This proves that the performance of the ANN-ICA model is better and more reliable in predicting slope stability behavior. In another study, Goh et al. [12] developed an ANN model with a configuration of 7-36-1 to predict maximum soil sedimentation. The trial and error methods are used to determine the number of hidden layered neurons. The ANN model's performance was validated using data from element analysis to and with data measured in the field. As a result, the ANN model developed can accurately predict. This indicates an optimal network structure capable of reading data patterns well.

Table 9 Performance indices of ANN [28]

\begin{tabular}{lllll}
\hline Model & $\mathbf{R}^{2}$ & RMSE & MAPE & VAF[\%] \\
\hline ANN-MLP & 0.942 & 0.83 & 13.6 & 94.11 \\
\hline ANN-RBF & 0.946 & 0.79 & 12.4 & 94.55 \\
\hline
\end{tabular}

Table 10 Performance indices of ANN models [30]

\begin{tabular}{lllll}
\hline Data & $\mathbf{R}^{2}$ & RMSE & MAE & VAF[\%] \\
\hline Training set & 0.986 & 0.13 & 0.10 & 98.67 \\
\hline Testing set & 0.986 & 013 & 0.10 & 98.60 \\
\hline Validation set & 0.985 & 0.13 & 0.11 & 98.50 \\
\hline
\end{tabular}

\section{Conclusions and future perspectives}

Many research studies have been published on forecasting road embankment stability using ANN by researchers worldwide. However, the publication of studies on embankment stability due to earthquake impacts using ANN remains limited. This paper reviews the importance of predictive research studies on road embankment stability using ANN. A review of 28 research papers shows that the ANN approach has been successfully applied in modeling and 315 predicting embankment stability with acceptable precision compared to some other models. Although there are some limitations in the ANN model, such as network configuration optimization, extrapolation errors, and overtraining errors, it is still widely used due to its simplicity. Besides, a comprehensive predicting system for road embankment stability, slope stability, settlement, geotechnical parameters, and seismic effects is not yet any researcher developing. This provides an opportunity for 
researchers to use the expert system as an AI to develop a prediction system. ANN's proposed future research on fortress stability is as follows:

i. To consider the ability of predictive models such as RBF, convolutional neural network (CNN), recurrent evolutionary neural network (RNN), deep neural network (DNN), extreme learning machine (ELM).

ii. Hybrid predictive methods, namely the hybrid radial basis function neural networks (HyRBF), hybrid deep neural network (HyDNN), and hybrid extreme learning machine (Hyelm), can be potentially used to produce more accurate results.

iii. Studies on optimization algorithms combined with ANN approaches are limited. Therefore, there is research needs to expand network configuration optimization using evolutionary computation methods such as genetic algorithm (GA), particle swarm optimization (PSO), artificial bee colony (ABC), and gravitational search algorithm (GSA).

iv. Predictive studies on road embankment stability supported by prefabricated vertical drains (PVDs) are too limited and can be considered for future research by using ANN and extended to methods such as support vector machine (SVM), response surface methodology (RSM), and adaptive neurofuzzy inference systems (ANFIS).

v. Measured data should be compared in advance with the output from limit equilibrium (LE) or finite element (FE) calculated to assess embankment stability with a low error before performing predictive ANN evaluations

vi. There is very limited literature on the global factor of safety assessment considerations, maximum base settlement, maximum surface settlement, and maximum deflection on the road embankment stability in design calculations before developing predictive models such as ANN.

Based on the reviews and discussions presented in this paper, it is concluded that the ANN method is a good model and can be used to predict road embankment stability. The results and information presented are beneficial to researchers who are conducting research using ANN in this field.

\section{Acknowledgment}

None.

Conflicts of interest

The authors have no conflicts of interest to declare.

\section{References}

[1] Che Mamat R. Engineering properties of Batu pahat soft clay stabilized with lime, cement and bentonite for subgrade in road construction (Doctoral dissertation, Universiti Tun Hussein Onn Malaysia). 2013: 1-129.

[2] Mamat RC, Ramli A, Samad AM, Kasa A, Razali SF, Omar MC. Stability assessment of embankment on soft soil improved with prefabricated vertical drains using empirical and limit equilibrium approaches. International Journal of Advanced Trends in Computer Science and Engineering. 2019; 8(1.6 Sp):444-9.

[3] Chik Z, Aljanabi QA, Kasa A, Taha MR. Tenfold cross validation artificial neural network modeling of the settlement behavior of a stone column under a highway embankment. Arabian Journal of Geosciences. 2014; 7(11):4877-87.

[4] Kanayama M, Rohe A, van Paassen LA. Using and improving neural network models for ground settlement prediction. Geotechnical and Geological Engineering. 2014; 32(3):687-97.

[5] Chik Z, Aljanabi QA. Intelligent prediction of settlement ratio for soft clay with stone columns using embankment improvement techniques. Neural Computing and Applications. 2014; 25(1):73-82.

[6] Bi QT, Ding SY. Neural networks model for settlement prediction of embankment. In applied mechanics and materials 2012 (pp. 722-6). Trans Tech Publications Ltd.

[7] Li XY, Bu FJ. Prediction of settlement of soft clay foundation in highway using artifical neural networks. In advanced materials research 2012 (pp. 15-20). Trans Tech Publications Ltd.

[8] Wang ZL, Li YC, Shen RF. Correction of soil parameters in calculation of embankment settlement using a BP network back-analysis model. Engineering Geology. 2007; 91(2-4):168-77.

[9] Hasanzadehshooiili H, Mahinroosta R, Lakirouhani A, Oshtaghi V. Using artificial neural network (ANN) in prediction of collapse settlements of sandy gravels. Arabian Journal of Geosciences. 2014; 7(6):2303-14.

[10] Kurnaz TF, Dagdeviren U, Yildiz M, Ozkan O. Prediction of compressibility parameters of the soils using artificial neural network. SpringerPlus. 2016; 5(1):1-11.

[11] Dinçer İ. Models to predict the deformation modulus and the coefficient of subgrade reaction for earth filling structures. Advances in Engineering Software. 2011; 42(4):160-71.

[12] Goh AT, Zhang RH, Wang W, Wang L, Liu HL, Zhang WG. Numerical study of the effects of groundwater drawdown on ground settlement for excavation in residual soils. Acta Geotechnica. 2020; 15(5):1259-72.

[13] Chok YH, Jaksa MB, Kaggwa WS, Griffiths DV, Fenton GA. Neural network prediction of the reliability of heterogeneous cohesive slopes. International Journal for Numerical and Analytical Methods in Geomechanics. 2016; 40(11):1556-69. 
[14] Sakellariou MG, Ferentinou MD. A study of slope stability prediction using neural networks. Geotechnical \& Geological Engineering. 2005; 23(4):419-45.

[15] Mamat RC, Kasa A, Razali SF, Ramli A, Omar MB. Slope stability prediction of road embankment on soft ground treated with prefabricated vertical drains using artificial neural network. IAES International Journal of Artificial Intelligence. 2020; 9(2):236-43.

[16] Erzin Y, Cetin T. The prediction of the critical factor of safety of homogeneous finite slopes using neural networks and multiple regressions. Computers \& Geosciences. 2013; 51:305-13.

[17] Erzin Y, Cetin T. The prediction of the critical factor of safety of homogeneous finite slopes subjected to earthquake forces using neural networks and multiple regressions. Geomechanics \& Engineering. 2014; 6(1):1-5.

[18] Abdalla JA, Attom MF, Hawileh R. Prediction of minimum factor of safety against slope failure in clayey soils using artificial neural network. Environmental Earth Sciences. 2015; 73(9):5463-77.

[19] Verma AK, Singh TN, Chauhan NK, Sarkar K. A hybrid FEM-ANN approach for slope instability prediction. Journal of The Institution of Engineers (India): Series A. 2016; 97(3):171-80.

[20] Gao W, Raftari M, Rashid AS, Mu'azu MA, Jusoh WA. A predictive model based on an optimized ANN combined with ICA for predicting the stability of slopes. Engineering with Computers. 2020; 36(1):32544.

[21] Kayadelen C, Günaydın O, Fener M, Demir A, Özvan A. Modeling of the angle of shearing resistance of soils using soft computing systems. Expert Systems with Applications. 2009; 36(9):11814-26.

[22] Park HI. Development of neural network model to estimate the permeability coefficient of soils. Marine Georesources \& Geotechnology.2011; 29(4):267-78.

[23] Islam SM, Chik Z, Mustafa M, Sanusi H. Model with artificial neural network to predict the relationship between the soil resistivity and dry density of compacted soil. Journal of Intelligent \& Fuzzy Systems. 2013; 25(2):351-7.

[24] Viji VK, Lissy KF, Sobha C, Benny MA. Predictions on compaction characteristics of fly ashes using regression analysis and artificial neural network analysis. International Journal of Geotechnical Engineering. 2013; 7(3):282-91.

[25] Kanungo DP, Sharma S, Pain A. Artificial neural network (ANN) and regression tree (CART) applications for the indirect estimation of unsaturated soil shear strength parameters. Frontiers of Earth Science. 2014; 8(3):439-56.

[26] Khanlari GR, Heidari M, Momeni AA, Abdilor Y. Prediction of shear strength parameters of soils using artificial neural networks and multivariate regression methods. Engineering Geology. 2012; 131:11-8.

[27] Ghorbani A, Hasanzadehshooiili H. Prediction of UCS and CBR of microsilica-lime stabilized sulfate silty sand using ANN and EPR models; application to the deep soil mixing. Soils and Foundations. 2018; 58(1):34-49.

[28] Yilmaz I, Kaynar O. Multiple regression, ANN (RBF, MLP) and ANFIS models for prediction of swell potential of clayey soils. Expert Systems with Applications. 2011; 38(5):5958-66.

[29] Gordan B, Armaghani DJ, Hajihassani M, Monjezi M. Prediction of seismic slope stability through combination of particle swarm optimization and neural network. Engineering with Computers. 2016; 32(1):85-97.

[30] Erzin Y, Cetin T. The use of neural networks for the prediction of the critical factor of safety of an artificial slope subjected to earthquake forces. Scientia Iranica. 2012; 19(2):188-94.

[31] Mamat RC, Samad AM, Kasa A, Razali SF, Ramli A, Omar MB. Slope stability analysis of road embankment on soft ground treated with prefabricated vertical drains. International Journal of Advanced Science and Technology. 2020; 29(6 Spec):834-42.

[32] Chan KF, Poon BM, Perera D. Prediction of embankment performance using numerical analysesPractitioner's approach. Computers and Geotechnics. 2018; 93:163-77.

[33] Paliwal M, Kumar UA. Neural networks and statistical techniques: a review of applications. Expert systems with applications. 2009; 36(1):2-17.

[34] Tang XW, Bai X, Hu JL, Qiu JN. Assessment of liquefaction-induced hazards using Bayesian networks based on standard penetration test data. Natural Hazards and Earth System Sciences. 2018; 18(5):1451-68.

[35] Kok BV, Yilmaz M, Sengoz B, Sengur A, Avci E. Investigation of complex modulus of base and SBS modified bitumen with artificial neural networks. Expert Systems with Applications. 2010; 37(12):777580.

[36] Biglarijoo N, Mirbagheri SA, Bagheri M, Ehteshami M. Assessment of effective parameters in landfill leachate treatment and optimization of the process using neural network, genetic algorithm and response surface methodology. Process Safety and Environmental Protection. 2017; 106:89-103.

[37] Yildirim B, Gunaydin O. Estimation of california bearing ratio by using soft computing systems. Expert Systems with Applications. 2011; 38(5):6381-91.

[38] Chakraborty A, Goswami D. Prediction of slope stability using multiple linear regression (MLR) and artificial neural network (ANN). Arabian Journal of Geosciences. 2017; 10(17):1-11.

[39] Kaboodvandpour S, Amanollahi J, Qhavami S, Mohammadi B. Assessing the accuracy of multiple regressions, ANFIS, and ANN models in predicting dust storm occurrences in Sanandaj, Iran. Natural Hazards. 2015; 78(2):879-93.

[40] Chou JS, Lin CW, Pham AD, Shao JY. Optimized artificial intelligence models for predicting project award price. Automation in Construction. 2015; 54:106-15. 
[41] Graupe D. Principles of artificial neural networks. World Scientific; 2013.

[42] Fyfe C. Artificial neural networks and information theory. Department of Computing and Information System, the University of Paisley. 2000.

[43] Orbanić P, Fajdiga M. A neural network approach to describing the fretting fatigue in aluminium-steel couplings. International Journal of Fatigue. 2003; 25(3):201-7.

[44] Haykin S, Network N. A comprehensive foundation. Neural Networks. 2004; 2(2004):41.

[45] Ashrafi HR, Jalal M, Garmsiri K. Prediction of loaddisplacement curve of concrete reinforced by composite fibers (steel and polymeric) using artificial neural network. Expert Systems with Applications. 2010; 37(12):7663-8.

[46] Das SK, Biswal RK, Sivakugan N, Das B. Classification of slopes and prediction of factor of safety using differential evolution neural networks. Environmental Earth Sciences. 2011; 64(1):201-10.

[47] Mamat RC, Kasa A, Razali SF. The applications and future perspectives of adaptive neuro-fuzzy inference system in road embankment stability. Journal of Engineering Science \& Technology Review. 2019; 12(5):75-90.

[48] Afan HA, El-Shafie A, Yaseen ZM, Hameed MM, Mohtar WH, Hussain A. ANN based sediment prediction model utilizing different input scenarios. Water Resources Management. 2015; 29(4):1231-45.

[49] He W, Chen Y, Yin Z. Adaptive neural network control of an uncertain robot with full-state constraints. IEEE Transactions on Cybernetics. 2015; 46(3):620-9.

[50] Esteva A, Kuprel B, Novoa RA, Ko J, Swetter SM, Blau HM, Thrun S. Dermatologist-level classification of skin cancer with deep neural networks. Nature. 2017; 542(7639):115-8.

[51] Raghu S, Sriraam N. Optimal configuration of multilayer perceptron neural network classifier for recognition of intracranial epileptic seizures. Expert Systems with Applications. 2017; 89:205-21.

[52] Beşikçi EB, Arslan O, Turan O, Ölçer AI. An artificial neural network based decision support system for energy efficient ship operations. Computers \& Operations Research. 2016; 66:393-401.

[53] Mirjalili S. How effective is the Grey Wolf optimizer in training multi-layer perceptrons. Applied Intelligence. 2015; 43(1):150-61.

[54] Lee S, Choeh JY. Predicting the helpfulness of online reviews using multilayer perceptron neural networks. Expert Systems with Applications. 2014; 41(6):30416.
[55] Mehrabi S, Maghsoudloo M, Arabalibeik H, Noormand R, Nozari Y. Application of multilayer perceptron and radial basis function neural networks in differentiating between chronic obstructive pulmonary and congestive heart failure diseases. Expert Systems with Applications. 2009; 36(3):69569.

[56] Chandwani V, Agrawal V, Nagar R, Singh S. Modeling slump of ready mix concrete using artificial neural network. International Journal of Technology. 2015; 6(2):207-16.

[57] Başyigit C, Akkurt I, Kilincarslan S, Beycioglu A. Prediction of compressive strength of heavyweight concrete by ANN and FL models. Neural Computing and Applications. 2010; 19(4):507-13.

[58] Amirian E, Leung JY, Zanon S, Dzurman P. Integrated cluster analysis and artificial neural network modeling for steam-assisted gravity drainage performance prediction in heterogeneous reservoirs. Expert Systems with Applications. 2015; 42(2):72340.

[59] Jamli MR, Ariffin AK, Wahab DA. Incorporating feedforward neural network within finite element analysis for L-bending springback prediction. Expert Systems with Applications. 2015; 42(5):2604-14.

[60] Mamat RC, Kasa A, Razali SM. Comparative analysis of settlement and pore water pressure of road embankment on yan soft soil treated with PVDs. Civil Engineering Journal. 2019; 5(7):1609-18.

[61] Uzlu E, Kankal M, Akpınar A, Dede T. Estimates of energy consumption in Turkey using neural networks with the teaching-learning-based optimization algorithm. Energy. 2014; 75:295-303.

[62] Mamat RC, Kasa A, Razali SF. A review of road embankment stability on soft ground: problems and future perspective. IIUM Engineering Journal. 2019; 20(2):32-56.

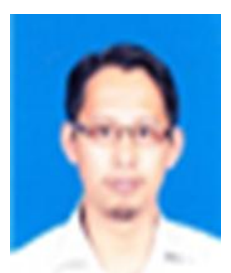

Rufaizal Che Mamat is a senior lecturer at Politeknik Ungku Omar and Politeknik Sultan Idris Shah. Also, he has served in Department of Polytechnic Education for 17 years. He began his undergraduate studies in 1998 at Universiti Teknologi Tun Hussien Onn, Malaysia. He is currently pursuing his Ph.D. at Universiti Kebangsaan Malaysia (UKM) in Geotechnical Engineering.

Email: rufaizal.cm@gmail.com 


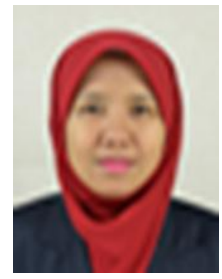

Azuin Ramli is a senior lecturer in Civil Engineering at Politeknik Ungku Omar (PUO). She received his doctorate from UTHM in 2015 in building construction. She has published over 12 articles in various fields of civil engineering.

Email: azuinramli@gmail.com

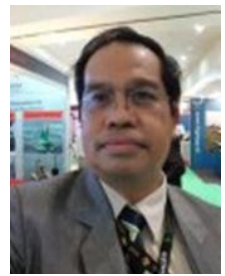

Abd Manan Samad is a professor at the Faculty of Architecture, Planning \& Surveying, Universiti Teknologi MARA (UiTM). He has served over 30 years in the education system. He has extensive experience in Land Survey Engineering research and has published over 350 articles.

Email: dr_abdmanansamad@ieee.org

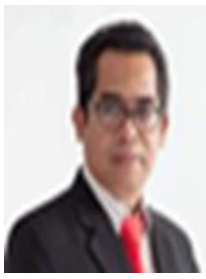

Anuar Kasa is a senior lecturer in Geotechnical Engineering at Universiti Kebangsaan Malaysia (UKM). He is also a professional engineer registered with the Board of Engineers Malaysia (BEM). He has published over 60 articles in various fields of Geotechnical Engineering.

Email: anuar@eng.ukm.my

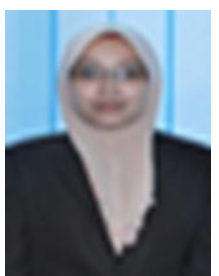

Siti Fatin Mohd Razali is an associate professor in water resources engineering at Universiti Kebangsaan Malaysia (UKM). She is also a professional engineer registered with the Board of Engineers Malaysia (BEM). She has published over 35 articles in various fields of Water Resources Engineering. Email: fatinrazali@ukm.edu.my

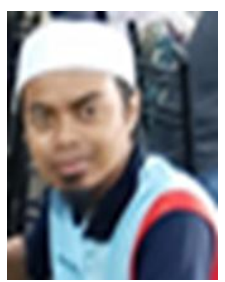

Mohd Badrul Hafiz Che Omar is a research assistant and is currently studying for a Ph.D. at Universiti Teknologi MARA (UiTM). $\mathrm{He}$ is currently conducting a research project in slope stability for an embankment on soft ground.

Email:2019563007@ siswa.uitm.edu.my 